\title{
Prácticas de crianza tradicionales, estilos de crianza parentales de riesgo en madres con hijos adolescentes
}

\author{
Traditional parenting practices, risky parenting styles \\ in mothers towards adolescent children
}

\section{Diana Lucia Pérez Santiago,I Jorge Armando Álzate Cataño,"II Miguel Ángel Cardona Valencia,,III Yuli Andrea Méndez HerreraIV}

I Psicóloga (Universidad Nacional Abierta y a Distancia, UNAD, Colombia). Candidata a magíster en Psicología Clínica y de la Salud (UNMSM, Perú). Docente e investigadora del Programa de Psicología de la Fundación Universitaria Católica del Norte, Medellín, Colombia). $\varangle \cdot d l p e r e z @ u c n . e d u . c o$ https://orcid.org/0000-0001-9801-6948

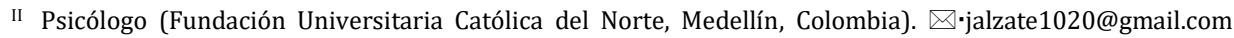
https://orcid.org/0000-0002-5338-0997

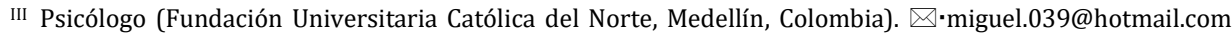
https://orcid.org/0000-0002-9592-426x

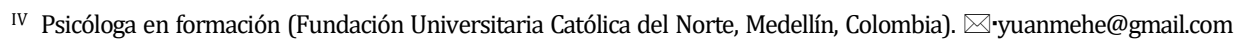
https://orcid.org/0000-0003-2344-9612

RECIBIDO: 30.10 .2019

ACEPTADO: 12.5 .2020

\section{Resumen}

El presente estudio tuvo como propósito analizar las creencias y actitudes de 15 madres de familia del municipio de Pensilvania (departamento de Caldas, Colombia), en su contexto familiar primario, sobre las prácticas de crianza tradicionales asociadas con los estilos de crianza parentales de riesgo que ejercen con sus hijos en edades entre los 12 y 16 años. La investigación se orientó según un tipo de estudio exploratorio-descriptivo, cuyo instrumento de recolección de información fue una guía de entrevista de grupos focales. Para la selección de la muestra poblacional se implementó el muestreo por 
conveniencia, y como procedimiento de análisis de datos se usó el método de análisis de datos cualitativos. En los resultados se evidenció que los comportamientos agresivos a nivel psicofísico ejercidos por las madres hacia sus hijos son una construcción multigeneracional consolidada desde las tradiciones culturales, que afectan negativamente la percepción del adolescente sobre sí mismo y sobre su mundo. Se concluye que el componente de crianza tradicional sociofamiliar influye de manera significativa en las creencias y actitudes maternales que expresan y practican en la crianza de sus hijos al momento de consolidar sus roles a nivel sociofamiliar.

Palabras clave: crianza del niño, actitud de los padres, papel de los padres, madre

\section{Abstract}

The purpose of this study was to analyze the beliefs and attitudes of 15 mothers about traditional parenting practices in their primary family context associated with risky parenting styles that they exercise with their children between the ages of 12 and 16 years of age. Municipality of Pensilvania-Caldas. This research was oriented with a type of exploratory-descriptive study, with the focus group interview guide being the information gathering instrument. For the selection of the population sample, convenience sampling was implemented, and as a data analysis procedure, the qualitative data analysis method was used. The results showed that the aggressive behaviors at the psychophysical level exercised by mothers towards their children are a consolidated multigenerational construction from existing cultural traditions, negatively affecting the adolescent's perception of herself and her world. It is concluded that the traditional socio-family upbringing component does significantly influence the maternal beliefs and attitudes that they express and practice in raising their children when consolidating their roles at the socio-family level.

Keywords: child rearing, parent attitudes, parent role, mothers.

\section{Introducción}

La presente investigación pretende ser parte de la contribución que se ha generado desde diferentes perspectivas de estudio a nivel mundial y nacional con relación a la influencia de las prácticas de crianza tradicionales de la familia de origen para el desarrollo de estilos de crianza parentales de riesgo en madres de familia con hijos adolescentes. Esta problemática se dinamiza al interior de las relaciones familiares entre padre, madre e hijos, en este caso, especialmente desde la mirada de la madre de familia cuando ejerce su rol para armonizar y alinear las conductas de los hijos, según su percepción del mundo, es decir, de lo que ella o ellas valoran o consideran que es importante inculcar en sus hijos adolescentes. Así como lo refiere Ramírez (2005), «los padres 
ponen en práctica unas tácticas llamadas estilos educativos, prácticas de crianza o estrategias de socialización con la finalidad de influir, educar y orientar a los hijos para su integración social» (párr. 5).

Por lo tanto, abordar el tema de estilos de crianza parentales es ingresar a un mundo de interpretaciones y asociaciones sobre la realidad que se teje y se cataloga al interior del funcionamiento familiar, pero que, para este estudio, se orienta a partir de lo que señala Comellas (citado por Capano y Ubach, 2013, p. 87) como «la forma de actuar, derivada de unos criterios, que identifica las respuestas que los adultos dan a los menores ante cualquier situación cotidiana, toma de decisiones o actuaciones».

En tal sentido, los padres y madres de familia pretenden tener la plena seguridad que con sus actos educativos aportan positivamente en la formación integral de sus hijos conforme al mundo actual, puesto que sus ideales de crianza asertiva se rigen bajo la premisa de que así han sido educados o formados desde sus contextos sociofamiliares de origen, los cuales les han proporcionado experiencias significativas en su vida, posibilitando ser lo que son ahora, personas honorables y disciplinadas.

Cabe resaltar que la Organización Panamericana de la Salud (OPS) señaló, en 2009, que «existe evidencia del rol de la familia y de la relación de los adolescentes con un adulto significativo como un factor crítico para su desarrollo saludable» (p. 8). Es importante señalar que en Colombia se registran estudios cercanos a la problemática, pero no con tanto interés desde el ámbito de la psicología sino de áreas afines como la sociología, pedagogía, antropología, derecho, etcétera, donde se resalta la influencia de las tradiciones socioculturales como un factor influyente dentro del sistema familiar. Así, según Gallego (2012), «como institución social, las familias se encuentran permeadas por una serie de factores sociales, políticos, estructurales e ideológicos que las atraviesan e inciden en las relaciones que se crean en su interior, como las prácticas de crianza» (p. 67).

Desde esta perspectiva, se pretende que esta investigación proporcione un conocimiento actualizado sobre el fenómeno de interés, y que contribuya a los procesos de atención e intervención desde la psicología con relación al funcionamiento familiar, puesto que, a pesar de que existen investigaciones relacionadas a evidenciar la influencia de las prácticas sociales y familiares en el mantenimiento de conductas inadecuadas en población infantil y adolescente, aún no se logra identificar exploraciones actualizadas que aborden el tema de la influencia de las tradiciones socioculturales y familiares en el desarrollo o mantenimiento de estilos de crianza parentales de riesgo en madres o padres de familia que afectan la forma de entender y de actuar de la población adolescente en un mundo aún diseñado para adultos.

A partir de lo anterior, es importante especificar que este estudio tuvo como objetivo principal el análisis de las creencias y actitudes de 15 madres de familia sobre las prácticas de crianza tradicionales en su contexto familiar primario asociadas con los estilos de crianza parentales de riesgo que ejercen con sus hijos en edades entre los 12 y 
16 años del municipio de Pensilvania (departamento de Caldas, Colombia). Así mismo, se generaron tres objetivos específicos orientados a:

- interpretar el significado que les atribuyen las madres de familia a las prácticas de crianzas tradicionales que se han ejercido en su contexto sociofamiliar de origen;

- describir las actitudes y creencias de riesgo presentes en madres de familia con relación a las prácticas de crianza que ejercen sobre sus hijos adolescentes; y

- señalar las afectaciones que generan en el comportamiento de los hijos los estilos de crianza inadecuados adoptados por las madres de familia con base en las tradiciones familiares o culturales de su entorno.

\section{Marco de referencia de investigación. Algunos antecedentes sobre prácticas de crianza tradicional y estilos de crianza parental}

Es relevante recordar que las pautas de crianza han sido tema de estudio que han sido abordados desde múltiples perspectivas de las ciencias sociales y humanas, permitiendo, entre otras, distinciones entre familia, prácticas, pautas y creencias, que de alguna manera han procurado comprender la dinámica del ser humano y sus manifestaciones en el entorno psicofamiliar.

De ese modo, se evidencian investigaciones en el ámbito internacional, nacional y local asociadas con alguna de las categorías de la problemática de interés. Así, se ubica en Costa Rica un artículo de Mariano Rosabal-Coto (2012), sobre creencias y prácticas de crianza, abordaje de parentaje, que brinda como resultado la necesidad de generar un abordaje más profundo sobre el parentaje, puesto que «la transmisión de la cultura está mediada no solo por la transmisión de creencias e ideas, sino también es adquirida a través de manifestaciones corporales y patrones interactivos (Keller et al., 2004; Rosabal-Coto, 2004)» (p. 94).

En este mismo orden de ideas, Torres et al. (2008) refuerzan lo anterior, a través de su investigación realizada en México, sobre responsabilidades en la crianza de los hijos, y concluyen que:

El ser padres (mamá o papá) es establecer una crianza medida por elementos educativos, de cuidado integral (socioemocional) de sus hijos, reconociendo el limitante que tiene todo padre o madre en las prácticas educativas que ejercen y que se adoptan de generación en generación con algunas adaptaciones a la época. (p. 86) 
Por otro lado, en Colombia, se resalta artículo de investigación científica y tecnológica resultado de la investigación «Pautas, creencias y prácticas de crianza relacionadas con el castigo y su transmisión generacional» realizada entre el 17 de enero de 2006 y el 9 de noviembre de 2007 por Sandra Pulido, Juliana Castro, Marlyn Peña y Diana Ariza, evidenciando que «las creencias se establecen en torno al niño o niña como fuerte y frágil, y al castigo como formador, ambivalente, maltratante y temido» (2013, p. 245).

\section{Marco teórico-conceptual. La familia: conceptualización y delimitación}

El Observatorio de Políticas de las Familias (OPF, 2015) refiere que se entiende por familia «el conjunto de personas entre las que median lazos cercanos de sangre, afinidad o adopción, independientemente de su cercanía física o geográfica y de su cercanía afectiva o emocional (Rubiano y Wartenberg, 1991)» (p. 9).

Bajo esta perspectiva, es necesario señalar que el concepto de familia ha sufrido importantes transformaciones a partir de eventos sociales, culturales, políticos, económicos y religiosos que han marcado huella en la historia de la vida humana, y que, por lo tanto, aún en la actualidad es complejo redefinir su delimitación conceptual, como lo menciona De León (2011):

Ahora, el delimitar el concepto de familia es algo complejo, porque es una estructura que dependiendo de la cultura en la que nos situemos y en la época en la que nos encontremos, va cambiando su estructura y va adaptándose a la realidad social en la que se encuentra inmersa. (p. 7)

En otro sentido, «la organización y función de las familias no solo cambia con los cambios sociales, culturales y económicos, sino que también depende de la etapa del ciclo de vida en que se encuentren» (OPF, 2015, p. 8). Esta situación genera asociaciones de interpretación variadas frente a la realidad que se experimenta al interior de la familia, en su dinámica de funciones y expectativas de vida, donde los estilos o prácticas crianza que ejercen los padres (madre y padre) sobre sus hijos van a marcar al individuo en su forma de interaccionar con los otros y el contexto donde se integre. Así lo refiere De León (2011):

Muchos autores parecen estar de acuerdo en señalar que la familia es para el individuo un sistema de participación, donde están expuestos a una serie de exigencias, un contexto donde se generan, expresan y se identifican las emociones, un 
entorno donde se promueven las primeras relaciones sociales, en el que se adquieren los valores que sustentan las acciones de las personas. (p. 7)

En esta perspectiva, la familia es el primer espacio para facilitar el desarrollo de habilidades sociales y de conductas prosociales en la infancia, que le permiten al niño, niña y/o adolescente adaptarse al contexto social donde interactúa, teniendo como base la importancia de la historia de la vida familiar primaria. Así mismo, es necesario indicar que, dentro de la tipología de estructura familiar, según OPF (2015), esta presenta unas particularidades, bajo las cuales se han clasificado. Es importante considerar que:

Se clasifica a los hogares con base en la relación de parentesco entre sus miembros con el jefe de hogar [...]. Con base en Ullmann, Maldonado Valera, y Rico (2014), se obtienen las siguientes categorías: hogares familiares (nucleares, amplios [extensos, compuestos] y familiares sin núcleo) y hogares no familiares (unipersonales y no familiares sin núcleo). (p. 10)

\section{La familia desde un enfoque sistémico}

Desde la perspectiva de la teoría familiar sistémica de Bowen, la familia requiere ser comprendida no solamente desde las formas presentes de interacción que se dan entre los individuos que la conforman, sino que también es vital reconocerla y comprenderla desde los eventos históricos y socioculturales que se tejen de una generación a otra, así como lo señala Bowen (citado por Ibáñez et al., 2017): «el comportamiento humano solo puede entenderse si se consideran también las dinámicas multigeneracionales de la familia del individuo» (p. 721). Por su parte, Rodríguez (2014), señala que:

Bowen considera al ser humano como la forma de vida más compleja, por ello se afirmará que gran parte de su funcionamiento humano está determinado por los mismos principios naturales presentes en otras formas de vida, con las que comparten procesos y mecanismos psíquicos, en particular en lo referente a la esfera emocional. (p. 4)

Es conveniente señalar que, desde la cosmovisión teórica de la familia que posee este autor, las relaciones familiares estarían permeadas por fuerzas vitales como son la autonomía y la vinculación, y que «ambas fuerzas están enraizadas en elementos instintivos, innatos y propios del funcionamiento del sistema emocional [...]. El equilibrio entre la autonomía y la vinculación dará como resultado unas relaciones familiares funcionales, con una adaptación saludable a las circunstancias» (Rodríguez, 2014, p. 4). 


\section{Estilos de crianza}

Con relación a los estilos de crianza existe una variedad de teorías en la actualidad que hacen posible la comprensión de la problemática que se dinamiza al interior de la vida familiar. Así, los estilos de crianza parentales son definidos como «esquemas prácticos que reducen las múltiples y minuciosas prácticas educativas paternas a unas pocas dimensiones, que, cruzadas [sic] entre sí en diferentes combinaciones, dan lugar a diversos tipos habituales de educación familiar» (Coloma, citado por Torio et al., 2008, p. 156).

Es necesario considerar que cada red familiar primaria recrea y formaliza atributos únicos que orientan sus acciones educativas sobre sus hijos, es decir, ideales sobre la dinámica de crianza que se precisa llevar en el ambiente familiar y que van alineados a las exigencias de su contexto cultural donde se incorporan o habitan. De ahí la importancia que resaltan Torio et al. (2008) al indicar que «no se identifican modelos puros, sino que se solapan, ya que los estilos educativos suelen ser mixtos y varían con el desarrollo del niño, no siendo estables a lo largo del tiempo» (p. 156).

Se puede dilucidar entonces que los vínculos de interacción que se dan entre padres e hijos son dinámicos, cambiantes, es decir, no son pasivos; esto conllevaría cambios significativos en la postura que asumen los actores involucrados en la dinámica familiar, en otras palabras, de padres a hijos, como lo señala Sánchez (2013):

El cuidado y el acompañamiento de los adultos en la educación debería ser una combinación equilibrada entre la ternura y el rigor, pues no podemos esperar que solo por los buenos sentimientos, por la sensibilidad que les podamos transmitir, por la nuestra capacidad de seducción, los hijos vayan a estar dispuestos siempre a ir por el camino que les indicamos. (pp. 42-43)

En virtud de lo anterior, y resaltando los estudios realizados por Diana Baumrind sobre este fenómeno de interacción o socialización familiar por varias décadas, se logra establecer la existencia de tres estilos de crianza ejercidos por los padres de familia a saber, autoritario, democrático y permisivo, según Huamán (2016):

Más adelante, el modelo de Diana Baumrind, según Coloma (1993), fue reformulado por Eleanor Maccoby y John Martin en 1983, quienes, basándose en dos dimensiones, control y afecto, desglosaron del estilo permisivo, originalmente propuesto por Baumrind, un nuevo estilo denominado negligente, el cual se caracteriza por un bajo nivel en ambas dimensiones. De esta forma se llega al modelo más aceptado de los cuatro estilos parentales, autoritativo o democrático (exigente y 
receptivo), autoritario (exigente y no receptivo), permisivo o indulgente (receptivo y no exigente) y permisivo negligente (no exigente y no receptivo). (p. 37)

Conviene subrayar que cada estilo parental posee una serie de características que posibilitan visibilizar su implicancia en el proceso adaptativo de los hijos en su contexto sociofamiliar. Así, Sánchez (2013) expone una serie de consecuencias educativas que emergen a partir de la clasificación anterior:

a. En el estilo autoritario, «los hijos se sienten etiquetados como buenos o malos. La relación de los padres es de hostilidad y temor» (p. 43).

b. El estilo responsable «crea un clima de libertad dentro de unos límites. Los hijos reconocen la autoridad basada en el diálogo. En el estilo permisivo, los hijos son dependientes y egoístas» (p. 44).

c. Y en el estilo no implicado existe «dificultad de adaptación al medio escolar y a cualquier grupo no permisivo» (p. 45).

\section{Actitudes y creencias parentales}

En la actualidad, en el mundo científico existe una diversidad de significados y funciones atribuidos al término actitud. Algunos autores lo asociarían desde los procesos de creencias (cognitivos), el valor (afectivos) y de predisposición para actuar (conativovolitivo) según el contexto, así como lo exterioriza Laca (2005) en su análisis del concepto, como «una respuesta implícita que tan solo aguarda la presencia de la situación estimuladora para pasar a ser respuesta manifiesta» (p. 119), o en su efecto como «manifestaciones de la experiencia consciente y necesitan de estímulos socioculturales» (Saavedra, 2018, p. 21). En otras palabras, «una preconducta, una disposición a actuar de terminada manera» (Díez, 2017, p. 138).

Esto implicaría anudar el elemento evaluativo, como lo evidencian Morales, Rebollo y Moya (1994, p. 497), citados por Mula y Navas (2013):

Resaltan la función de evaluación del objeto de actitud, entendiendo como evaluación «el efecto que despierta, las emociones que moviliza, el recuerdo emotivo de las experiencias vividas, incluso las creencias acerca de la capacidad del objeto para conseguir metas deseadas» y sostienen que en el proceso de evaluación influyen, con distinta intensidad, procesos cognitivos, afectivos y conductuales. (p. 17)

Las actitudes, así pues, posibilitan satisfacer la necesidad básica de conocimiento y autoridad con relación al contexto donde se relaciona el ser humano, puesto que 
facilitan estructurar la información que se percibe de la realidad. De ese modo, «Rauh, Wasserman y Brunelli (1990) consideran determinantes las actitudes maternales en las prácticas de crianza, puesto que estarían implicadas con el proceso de aculturación» (Ramírez, 2005, pp. 168-169).

La relación entre madre e hijo se encuentra mediada por unas estrategias de socialización, las cuales se han formado o consolidado a través de las experiencias de vida del padre o madre a partir de su red primaria, es decir, a partir de sus creencias, que se manifiestan a través de las actitudes que asumen y presentan ante los demás. Dentro de este marco, la teoría de la acción razonada, modelo propuesto por Fishbein y Ajzen (1980, citado por Reyes, 2007),

[...] es sumamente completo y permite obtener mayor seguridad en la medición de los factores determinantes de la conducta [...]. La teoría de la acción razonada consiste en elaborar un índice de probabilidad de la intención hacia la conducta que relaciona la actitud con las creencias del individuo. (p. 66)

En efecto, la comprensión de cómo se forman las creencias a partir de este modelo, teorizado a partir de tres tipos de creencias — descriptivas, inferenciales y formativas-, posibilita una vía para la explicación de la formación de las actitudes, las cuales están vinculadas por la fuerza de la creencia sobre el objeto de evaluación; en otras palabras, «la evaluación del atributo contribuye a definir la actitud del individuo en proporción a la fuerza de sus creencias» (Reyes, 2007, p. 68).

\section{Método}

Con la intención de ampliar la información obtenida para esta investigación, se sustentó un nivel de profundidad exploratorio-descriptivo y con un diseño situacional de campo, que posibilitó indagar con la técnica de grupo focal (guía de entrevista) las creencias y actitudes que emergen de las prácticas de crianza tradicional asociadas con los estilos de crianza de riesgo que ejercen las madres de familia sobre sus hijos adolescentes.

\section{Población participante}

La muestra poblacional estuvo conformada por 15 madres de familia, elegidas sobre la perspectiva del muestreo no probabilístico y por conveniencia, el cual «se utiliza si el investigador necesita que los posibles participantes se presenten por sí mismos» 
(Salamanca y Crespo, 2007, p. 2), lo que dio lugar al involucramiento de familias pertenecientes del municipio de Pensilvania en Caldas, Colombia.

La edad de las participantes se ubicó entre los 30 y los 60 años, según tres sublíneas de categorización: un 65\% de ellas tenían entre 30 y 40 años; un 33\%, entre 41 y 50 años, y $2 \%$ entre 51 y 60 años.

Así mismo, un dato importante es que para un 53,33\% de las participantes su tipo de estructura familiar era monoparental, un 26,67\% tenían familia nuclear y un $2 \%$ tenían una estructura de familia extensa. Por otro lado, en la categorización de estado civil sobresale que nueve de ellas eran solteras, dos casadas, dos viudas, una separada yuna divorciada.

\section{Instrumentos}

Con el propósito de dinamizar los procesos de recolección de información, y considerando que la población objeto de estudio es una comunidad continuamente involucrada en procesos de indagación, desde diferentes actores sociales, por programas del Estado y ONG, se optó por usar la técnica de grupos focales, como una estrategia que posibilita que los integrantes que conforman un grupo puedan exponer con mayor apertura sus percepciones sobre el fenómeno de interés, así como lo exponen Kitzinger y Martínez (citados por Hamui-Sutton y Varela-Ruiz, 2013): «la técnica de grupos focales es un espacio de opinión para captar el sentir, pensar y vivir de los individuos, provocando autoexplicaciones para obtener datos cualitativos» (p. 56).

De ahí que la guía de entrevista de grupos focales se convirtió en el instrumento de recolección de datos apropiado para este estudio; para ello fue vital que, al momento de la construcción de las preguntas detonantes de la indagación subjetiva, estas fueran formuladas con claridad — al decir de Mella (2000, p. 21), «las palabras con las que se formule la pregunta tienen que ser claramente entendibles para los participantes»-.

\section{Procedimiento}

El estudio se realizó desde un contexto comunitario; se generaron procesos pertinentes de acercamiento previo a la población a través de la convocatoria inicial a un espacio de reunión de carácter informativo, donde se explicó el propósito y alcance de este estudio. Así mismo, se invitó a leer y firmar el consentimiento informado para participar en la investigación.

Posteriormente, se elaboró una agenda de reunión con las participantes de cada grupo focal (dos grupos), para establecer las fechas y horarios de reunión. De esa forma, 
se asignó a cada grupo dos sesiones de reunión durante el proceso de recolección de información.

\section{Técnica de análisis de información}

Para el análisis de información de las entrevistas de grupos focales se utilizó la técnica de análisis de contenido de datos cualitativos sobre las transcripciones de las entrevistas. De ese modo, el primer acercamiento analítico a la información recolectada permitió la categorización de las respuestas y, posteriormente, en un segundo acercamiento, se reconstruyeron o fortalecieron las unidades de análisis a partir de los argumentos que la integraron.

\section{Resultados}

A modo de perfil, la tabla 1 evidencia la mayoría de los componentes actitudinales principales que emergen de la evocación sobre prácticas de crianza tradicional de la familia de origen, donde el respeto a padres o figura de autoridad es considerado como parte fundamental para el proceso de adaptación y socialización de los hijos en los diferentes espacios donde se vinculan, desde la perspectiva de las madres de familia, puesto que «les han permitido establecer límites, normas y metas en su familia actual», creencia que deriva silogismos, es decir, que conduce a una serie de ideas argumentativas para determinar y explicar las acciones realizadas o por efectuar, derivando en este caso la adecuación o mantenimiento de varios elementos prácticos que constituyen el modelo de crianza familiar de origen asociado a su estilos de crianza actual. 
Tabla 1. Agrupación de temas, actitudes y creencias en madres de familia con prácticas de crianza tradicional, subcategoría 1

\begin{tabular}{|c|c|c|c|}
\hline \multirow{2}{*}{$\begin{array}{l}\text { Componente ac- } \\
\text { titudinal (TAR) } \\
\text { cognitivo. } \\
\text { Categoría } 1\end{array}$} & \multicolumn{3}{|c|}{ Creencias formadoras de actitud (TAR) } \\
\hline & C. Descriptivas & C. Inferenciales & C. Informativas \\
\hline $\begin{array}{l}\text { Respeto a pa- } \\
\text { dres o autoridad }\end{array}$ & $\begin{array}{l}\text { «Si lo mandaban a } \\
\text { uno, por algo es por } \\
\text { lo que era así; no co- } \\
\text { mo hoy en día: “¡ma- } \\
\text { mi, ya voy!”» (p. 9) }\end{array}$ & $\begin{array}{l}\text { «Yo soy la que } \\
\text { mando en mi casa» } \\
\text { (p. 4) }\end{array}$ & $\begin{array}{l}\text { «Yo pienso que las } \\
\text { creencias son como } \\
\text { las normas y los va- } \\
\text { lores» (p. 2) }\end{array}$ \\
\hline $\begin{array}{l}\text { Posiciónracional } \\
\text { frente a la } \\
\text { crianza }\end{array}$ & $\begin{array}{l}\text { «A mí sí me ha to- } \\
\text { cado, a mí me ha to- } \\
\text { cado duro, con mis } \\
\text { hijos, de ser de papá } \\
\text { y mamá por ellos» } \\
\text { (p. 15) }\end{array}$ & & $\begin{array}{l}\text { «Yo creo que son } \\
\text { como unas pautas, } \\
\text { unos límites, unos } \\
\text { modelos para uno } \\
\text { formar los hijos» (p. } \\
\text { 7) }\end{array}$ \\
\hline $\begin{array}{l}\text { Límites, normas, } \\
\text { valores y accio- } \\
\text { nes correctivas }\end{array}$ & $\begin{array}{l}\text { «Desde muy peque- } \\
\text { ñitos uno tiene que } \\
\text { inculcarles unos va- } \\
\text { lores, unas normas } \\
\text { con ellos, que si se } \\
\text { promete un castigo, } \\
\text { hay que cumplirles; } \\
\text { un premio también» } \\
\text { (p. 12) }\end{array}$ & $\begin{array}{l}\text { «En el caso mío, } \\
\text { cuando yo estaba en } \\
\text { mi casa había hora- } \\
\text { rio, unos sitios para } \\
\text { estar y que mis pa- } \\
\text { pás me estuvieran } \\
\text { vigilando; y, pues, } \\
\text { con mi hija ahora yo } \\
\text { también le digo que } \\
\text { donde ella se mueva } \\
\text { yo la pueda estar vi- } \\
\text { gilando» (p. } 7 \text { ) }\end{array}$ & $\begin{array}{l}\text { «A mí me parece que } \\
\text { los están malcriando } \\
\text { y lavando el cerebro } \\
\text { totalmente» (p. 2) } \\
\text { «Yo creo que tiene } \\
\text { que haber una com- } \\
\text { binación de las dos. } \\
\text { Dicen que todo tiem- } \\
\text { po pasado fue mejor, } \\
\text { pero si uno se va a } \\
\text { los golpes, eso creó } \\
\text { muchos traumas en } \\
\text { la gente» (p. 8) }\end{array}$ \\
\hline $\begin{array}{l}\text { Continuidad del } \\
\text { modelo de } \\
\text { crianza familiar }\end{array}$ & $\begin{array}{l}\text { «Yo digo, pues, que } \\
\text { las tradiciones pues } \\
\text { como que continúan; } \\
\text { yo crecí en una fami- } \\
\text { lia muy humilde, de- } \\
\text { masiado humilde. Mi } \\
\text { mama siempre nos } \\
\text { inculcó muchos valo- } \\
\text { res del respeto por } \\
\text { las personas, la res- } \\
\text { ponsabilidad de las } \\
\text { cosas que uno hace, } \\
\text { la honestidad; y, } \\
\text { pues, uno trata de } \\
\text { cómo seguir ese mis- } \\
\text { mo ejemplo» (p. 7) }\end{array}$ & $\begin{array}{l}\text { «Si a uno le inculca- } \\
\text { ron valores, uno } \\
\text { trata de inculcarlos a } \\
\text { los hijos también. En } \\
\text { mi casa lo que son } \\
\text { los valores, gracias a } \\
\text { mi Dios» (p. 13) }\end{array}$ & $\begin{array}{l}\text { «A nosotros sí nos } \\
\text { tocaba llegar a traba- } \\
\text { jar, porque había } \\
\text { finca. Yo digo que eso } \\
\text { es lo que está pa- } \\
\text { sando, yo tengo un } \\
\text { hijo de } 16 \text { años y } \\
\text { ahora él es el que } \\
\text { manda, eso está } \\
\text { grave» (p. 1) } \\
\text { «Mi mamá, pues, fue } \\
\text { una mujer dema- } \\
\text { siado dura. Mi mamá } \\
\text { nos castigó mucho y } \\
\text { mi papa no» (p. 15) }\end{array}$ \\
\hline
\end{tabular}

Fuente: Elaboración propia. 
Tabla 2. Agrupación de temas, actitudes y creencias de riesgo en madres de familia con prácticas de crianza tradicional, subcategoría 3

\begin{tabular}{|c|c|c|c|}
\hline \multirow{2}{*}{$\begin{array}{l}\text { Componente actitu- } \\
\text { dinal (TAR) afec- } \\
\text { tivo. Categoría } 3\end{array}$} & \multicolumn{3}{|c|}{ Creencias formadoras de actitud (TAR) } \\
\hline & C. Descriptivas & C. Inferenciales & C. Informativas \\
\hline $\begin{array}{l}\text { Desconfianza, } \\
\text { miedo }\end{array}$ & & $\begin{array}{l}\text { «Era una persecu- } \\
\text { ción, por lo menos, } \\
\text { mi papá se la pasaba } \\
\text { buscándonos todo el } \\
\text { tiempo por el pue- } \\
\text { blo» (pp. } 9 \text { y 5) }\end{array}$ & \\
\hline $\begin{array}{l}\text { Flexibilidad, } \\
\text { permisividad, } \\
\text { afecto }\end{array}$ & $\begin{array}{l}\text { «Entonces, si pasamos un } \\
\text { rato de sano esparci- } \\
\text { miento, chévere, comiendo } \\
\text { lo que a ellos les gusta, } \\
\text { pues no es lo habitual de la } \\
\text { casa sino otras cositas, en- } \\
\text { tonces ellos se sienten pre- } \\
\text { miados con esos estímu- } \\
\text { los» (pp. 12, 3 y 4) } \\
\text { «Que le acepten los ami- } \\
\text { guitos, no importa el ami- } \\
\text { guito, sino que ellos se } \\
\text { sienten estimulados por- } \\
\text { que le apreciamos el ami- } \\
\text { guito» (p. 11) }\end{array}$ & & \\
\hline $\begin{array}{l}\text { Amor, } \\
\text { espiritualidad }\end{array}$ & $\begin{array}{l}\text { «El mejor regalo que una } \\
\text { madre le puede dar a un } \\
\text { hijo es cada día bende- } \\
\text { cirlo» (p. 15) }\end{array}$ & $\begin{array}{l}\text { «Lo que quedó de mi } \\
\text { mamá es todo el } \\
\text { amor que yo les doy a } \\
\text { mis hijos, porque uno } \\
\text { da de lo que tiene, } \\
\text { uno da de lo que re- } \\
\text { cibe y lo único que yo } \\
\text { recibí en toda mi vida } \\
\text { con mi mamá fue } \\
\text { amor» (p. 8) }\end{array}$ & \\
\hline $\begin{array}{l}\text { Ausencia de } \\
\text { imagen materna, } \\
\text { valoración de } \\
\text { apoyo familiar. }\end{array}$ & $\begin{array}{l}\text { «Yo soy hija de una profe- } \\
\text { sora, mi mamá enseñaba } \\
\text { en el campo y mi abuelita } \\
\text { fue la que me levantó } \\
\text { aquí en el pueblo. Tenía } \\
\text { la tristeza de no estar con } \\
\text { mi mamá, y ahora yo soy } \\
\text { la que trabajo y ellos se } \\
\text { han tenido que criar so- } \\
\text { los, se repite la historia» } \\
\text { (p. 10) }\end{array}$ & & \\
\hline
\end{tabular}




\begin{tabular}{|c|c|}
\hline $\begin{array}{l}\text { Insatisfacción, } \\
\text { fracaso }\end{array}$ & $\begin{array}{l}\text { «Pues yo, por lo menos, } \\
\text { no he podido criar a mi } \\
\text { hijo de ninguna forma» } \\
\text { (p. } 1 \text { ) }\end{array}$ \\
\hline $\begin{array}{l}\text { Angustia } \\
\text { e impotencia }\end{array}$ & $\begin{array}{l}\text { «Siento impotencia para } \\
\text { corregirlo, ya que no } \\
\text { tengo autoridad sobre } \\
\text { él» (p. 13) }\end{array}$ \\
\hline $\begin{array}{l}\text { Comunicación, } \\
\text { asertividad, } \\
\text { manejo de roles }\end{array}$ & $\begin{array}{l}\text { «En mi caso, por lo me- } \\
\text { nos, yo soy la que medio } \\
\text { trata de conciliar, porque } \\
\text { él es muy duro, porque } \\
\text { yo soy la amorosa, la } \\
\text { comprensiva; pero, en sí, } \\
\text { funciona» (p. 7) }\end{array}$ \\
\hline
\end{tabular}

Fuente: Elaboración propia.

En la tabla 2 se aprecian algunas de las creencias ensambladas por las madres de familia a partir de los procesos de interacción familiar tradicional de origen, que evidencian las distorsiones que se generan en los procesos de adaptación de estilos de crianza parental intergeneracionales, actitudes sesgadas como desconfianza, temor, miedo, angustia, frente a las situaciones o eventos que se dan en las acciones correctivas frente a conductas de interacción familiar, especialmente entre madre e hijos adolescentes, fortaleciendo en la figura de autoridad madre, ideas o estrategias correctivas en ocasiones inapropiadas que generan conductas recíprocas de intimidación entre madre e hijos.

En la tabla 3 se observa el establecimiento de patrones de crianza asumidos como válidos por la figura materna, en este caso, madre de familia, para la toma de decisiones y el establecimiento de la autoridad en el hogar a través de la aceptación de las reglas (normas) por los hijos. Patrones de crianza que son derivación de los estilos de crianza generados en su red familiar primaria que, al consolidarse, posiblemente desde experiencias educativas difusas, no generan el efecto esperado y desencadenan situaciones de violencia psicofísica e incluso el establecimiento de una pobre comunicación entre madre e hijo, o de insatisfacción por el fracaso de las estrategias de crianza parental que conllevan la pérdida de validez de la mamá frente a su hijo: «siento impotencia para corregirlo, ya que no tengo autoridad sobre él» (p. 13).

Por otro lado, también se identifican sentimientos de gratitud y excusa frente a las acciones de sus padres, por ejemplo, cuando expresan «es que ellos eran personas sin estudio» (p. 4) o «bueno, los golpes me ayudaron a valorar lo que me daban mis padres» (p. 6) y, al mismo tiempo, se establecen patrones de reflexión crítica entre las 15 madres de familia frente a la crianza tradicional recibida y la que podrían ejercer algunas de ellas, repitiendo esos patrones comportamentales de riesgo sobre sus hijos «pero si uno se va a los golpes, eso crea muchos traumas en la gente» (p. 8). 
Tabla 3. Influencia de prácticas de crianza tradicional en la dinámica de integración de estrategias educativas de riesgo en madres de familia

\begin{tabular}{|c|c|c|c|}
\hline \multirow{2}{*}{$\begin{array}{l}\text { Componente ac- } \\
\text { titudinal (TAR) } \\
\text { conativo inten- } \\
\text { cional. } \\
\text { Subcategoría } 4\end{array}$} & \multicolumn{3}{|c|}{ Creencias formadoras de actitud (TAR) } \\
\hline & C. Descriptivas & C. Inferenciales & C. Informativas \\
\hline \multirow[t]{2}{*}{$\begin{array}{l}\text { Aceptación de las } \\
\text { reglas }\end{array}$} & $\begin{array}{l}\text { «n mi casa, mi } \\
\text { hijo cuando nece- } \\
\text { sita algo me marca } \\
\text { y yo también lo } \\
\text { hago, porque a ve- } \\
\text { ces no puedo subir }\end{array}$ & $\begin{array}{l}\text { «En mi casa les } \\
\text { pongo orden y hasta } \\
\text { que no terminen el } \\
\text { trabajo no me salen, } \\
\text { y hasta ahora me ha } \\
\text { ido bien» (p. 13) }\end{array}$ & \\
\hline & $\begin{array}{l}\text { y para no po- } \\
\text { nerme a gritar» } \\
\text { (p. 6) }\end{array}$ & $\begin{array}{l}\text { «Así me toca vol- } \\
\text { verme una fiera, me } \\
\text { hacen caso, porque } \\
\text { me hacen caso» (p. 6) }\end{array}$ & \\
\hline \multirow[t]{2}{*}{$\begin{array}{l}\text { Toma de } \\
\text { decisiones }\end{array}$} & $\begin{array}{l}\text { «Yo primeramente } \\
\text { miro y analizo a } \\
\text { ver si es verdad } \\
\text { que merece ser } \\
\text { premiado» (p. } 15 \text { ) }\end{array}$ & $\begin{array}{l}\text { «Yo poco a los gol- } \\
\text { pes; le deben sacar a } \\
\text { uno mucho la piedra } \\
\text { para darles uno; } \\
\text { pero de por sí son } \\
\text { útiles, porque uno }\end{array}$ & $\begin{array}{l}\text { «Sí, yo reprendía, o } \\
\text { sea, la técnica que } \\
\text { yo le daba hasta los } \\
14 \text { años; ya, luego, } \\
\text { dialogar mucho con } \\
\text { ella» (p. 13) }\end{array}$ \\
\hline & & $\begin{array}{l}\text { les exige respeto y } \\
\text { uno tiene que darlo» } \\
\text { (p. 9) }\end{array}$ & $\begin{array}{l}\text { «Uno estimularlos y } \\
\text { felicitarlos por el } \\
\text { buen comporta- } \\
\text { miento y si se les } \\
\text { prometió algo cum- } \\
\text { plírselos, pues debe } \\
\text { de cumplírselos» } \\
\text { (p. } 10 \text { ) }\end{array}$ \\
\hline
\end{tabular}

Fuente: Elaboración propia.

En la misma línea, y con base en la tabla 4, también se resalta desde el componente conativo-intencional y comportamental la influencia de experiencias de prácticas de crianza familiar primaria tradicional en la dinámica de integración de estrategias educativas de riesgo en madres de familia, donde el ejercicio de poder y control sobre las acciones actitudinales inapropiadas que manifiestan sus hijos adolescentes en los diferentes espacios de interacción social reviven o promueven en la madre de familia la toma de decisiones erráticas con relación al estilo de crianza parental que necesita asumir y aplicar de forma asertiva con sus hijos adolescentes. 
Tabla 4. Consecuencias de estilos de crianza inadecuados ejercidos por la figura materna derivados de la familia de origen en el adolescente

\begin{tabular}{|c|c|c|c|}
\hline \multirow{2}{*}{$\begin{array}{l}\text { Componente } \\
\text { actitudinal } \\
\text { (TAR) com- } \\
\text { portamental }\end{array}$} & \multicolumn{3}{|c|}{ Creencias formadoras de actitud (TAR) } \\
\hline & C. Descriptivas & C. Inferenciales & C. Informativas \\
\hline $\begin{array}{l}\text { No hay más } \\
\text { opción que } \\
\text { obedecer }\end{array}$ & $\begin{array}{l}\text { «Uno obedecía, } \\
\text { ahora no obedecen, } \\
\text { los muchachos no le } \\
\text { obedecen a uno» } \\
\text { (p. 1) } \\
\text { «Cuando tenga mis } \\
\text { hijos, yo no les voy a } \\
\text { pegar y yo les doy a } \\
\text { todos, o sea, yo } \\
\text { pensé en mí, yo no } \\
\text { les voy a pegar, por- } \\
\text { que a mí me parecía } \\
\text { muy horrible» (p. 1) }\end{array}$ & $\begin{array}{l}\text { «La letra con sangre } \\
\text { entra» (p. 9) } \\
\text { «Yo, por ejemplo, } \\
\text { con los nietecitos, } \\
\text { cuando me toca ma- } \\
\text { nejarlos a mí, les } \\
\text { doy sus nalgaditas o } \\
\text { sus pertinacitos } \\
\text { (golpe con la pre- } \\
\text { tina o cinturón), } \\
\text { pues no todos los } \\
\text { días ni a toda hora» } \\
\text { (p. 10) } \\
\text { «En el caso mío, yo } \\
\text { nunca les prometí } \\
\text { nada, pues les hacía } \\
\text { cumplir las reglas» } \\
\text { (p. 11) }\end{array}$ & $\begin{array}{l}\text { «A mí me ha funcio- } \\
\text { nado otra cosa, que } \\
\text { tenía mi papá: es de } \\
\text { hablar y que se cum- } \\
\text { pla, es decir, lo hace } \\
\text { lo hace» (p. 4) } \\
\text { «Cuando vivía con mi } \\
\text { mamá y papá, ellos } \\
\text { daban una orden y de } \\
\text { inmediato se cum- } \\
\text { plía; a ahora ya no: se } \\
\text { da una orden a un } \\
\text { hijo y no la cumplen, } \\
\text { y si se les hace algo, } \\
\text { dicen que se van para } \\
\text { la comisaria y la van } \\
\text { demandar» (p. 1) } \\
\text { «Hoy en día se da eso } \\
\text { porque hay mucha } \\
\text { sobreprotección por } \\
\text { algunas entidades, } \\
\text { por eso los jóvenes } \\
\text { no quieren hacer } \\
\text { nada y dejan todo ti- } \\
\text { rado, en cambio, } \\
\text { ahora años nosotras } \\
\text { nos levantaron traba } \\
\text { jando y en orden» } \\
\text { (p. 15) }\end{array}$ \\
\hline
\end{tabular}

Fuente: Elaboración propia.

Esta situación predispone a la figura materna (madre) a asumir en muchas ocasiones un estado de invalidez, de baja asertividad frente a los procesos de crianza que ejerce frente a sus hijos, y conllevan a que manifieste actitudes violentas para ser escuchada y respetada, como lo señala una de las participantes: «yo poco a los golpes, le deben sacar a uno mucho la piedra para darles uno, pero de por sí son útiles, porque uno les exige respeto y uno tiene que darlo» (p. 9).

Al mismo tiempo se observa, con menor grado de participación en otro grupo de madres de familia, actitudes basadas en creencias de cambio con relación a la 
inefectividad de las estrategias o patrones de crianza asumidos o adoptados desde su red familiar primaria. Por ejemplo, una de las participantes expone que «sí, yo reprendía; o sea, la técnica que yo le daba hasta los 14 años; ya luego dialogar mucho con ella, porque lo anterior ya no funciona» (p. 13).

\section{Discusión y conclusiones}

Los resultados del presente trabajo son consistentes con otros estudios realizados por Infante y Martínez (2016, p. 31) los cuales señalan que el modelo parental se traduce en una pauta de crianza negociadora, un vínculo de apego cercano y un patrón de éxito promotor, así como Torres et al. (2008), donde se evidencia que el ser padres (mamá o papá) es establecer una crianza medida por elementos educativos, de cuidado integral (socioemocional) de sus hijos, reconociendo la limitación que tiene todo padre o madre en las prácticas educativas que ejerce, y que se adoptan de generación en generación con algunas adaptaciones a la época (p. 86).

Así, las creencias y actitudes de la madre de familia sobre el significado que le atribuye a las prácticas de crianza tradicional se direccionan al seguimiento de las normas establecidas desde la figura del padre o la madre, y se consolidan bajo el concepto de respeto a padres. Estas creencias han sido enraizadas desde las primeras experiencias de contacto normativo-afectivo al interior del hogar familiar primario, es decir, desde esa reconstrucción, aplicación y adecuación de los patrones de crianza multigeneracional que ha ido permeando la forma de ver, comprender e interactuar con la realidad.

Esto estaría encauzado propiamente sobre creencias inferenciales, que según Reyes (2007, citando a Ajzen y Fishbein) tienen su origen en las creencias descriptivas realizadas con antelación y se basan principalmente en relaciones probabilísticas entre las creencias, las cuales siguen los lineamientos del razonamiento silogístico (p. 68).

Otro hallazgo importante emerge en este estudio, direccionado a la evidencia de transformación o resignificación continua de las creencias formadoras de actitud con el ensamblaje de otras creencias que se han validado a partir de esas experiencias de vida inicial, y que se requieren usar para mitigar las expectativas de orientación de su entorno familiar actual, es decir, brota «una posición racional frente a lo que es la crianza tradicional recibida» interpretada como fuente de castigo-violencia, de afectividad y de éxito normativo al mismo tiempo, donde las acciones de castigo con violencia de sus progenitores es subsanada o excusada por las madres de familia bajo el concepto de gratitud, afecto y lealtad hacia sus padres, puesto que, a través de esas acciones correctivas y reconocidas como erradas o extremas, les permitieron ser las personas que son ahora. 
Situación anterior que predispone a la figura materna (madre) a asumir en varias ocasiones un estado de invalidez, de inasertividad frente a los procesos crianza que ejerce frente a sus hijos, conllevándola a manifestar actitudes de dos tipos: por un lado, y en menor escala, permisivas, y con mayor ponderación autoritarias violentas, con el único objetivo de ser escuchada y respetada, acciones que son interpretadas por el adolescente como patrones de crianza no comprensibles que le generan inestabilidad emocional para una adaptación relacional positiva entre pares y adultos; con base en ello se podría evidenciar en la mayoría de madres de familia un estilo de crianza parental autoritario, el cual se ha caracterizado por considerar la obediencia como una cualidad, así como lo sustentan Torio et al. (2008):

Los padres autoritarios (authoritarian discipline) valoran la obediencia como una virtud, así como la dedicación a las tareas marcadas, la tradición y la preservación del orden. Favorecen las medidas de castigo o de fuerza y están de acuerdo en mantener a los niños en un papel subordinado y en restringir su autonomía. (p. 158)

Anudado a lo anterior, Rodríguez (2014) resalta que las emociones tienen una influencia significativa en las creencias y acciones de todas las personas, ya que son útiles y necesarias para la adaptación al ambiente, puesto que son, de hecho, un producto evolutivo; por ello, el proceso de transmisión multigeneracional planteado por Bowen significa que el modo en que una familia maneja sus procesos emocionales se transmite de generación en generación y que, además, los procesos emocionales que percibimos en una familia nuclear están conectados con su familia extensa (p. 5).

Por consiguiente, y en la misma línea, Reyes (2007, p. 67) señala que la formación de una creencia implica la unión entre dos aspectos determinados del mundo de un individuo; esto tiene el propósito de conseguir el entendimiento de sí mismo y de su ambiente.

En concordancia con lo anterior, según Rodríguez (citado por Cuervo, 2010, p. 115), la familia es el primer contexto para la transmisión de las normas, valores y modelos de comportamiento. La familia es la que socializa al niño permitiéndole interiorizar los elementos básicos de la cultura y desarrollar las bases de su personalidad; cada familia asume las pautas de crianza dependiendo de sus características, dinámica y factores contextuales, así como de los recursos y apoyos, entre otros.

Bajo la misma perspectiva lo refiere De León (2011): «Muchos autores parecen estar de acuerdo en señalar que la familia es para el individuo un sistema de participación, [...] un entorno donde se promueven las primeras relaciones sociales, en el que se adquieren los valores que sustentan las acciones de las personas» (p. 7). 
Por ello, Sánchez (2011) señala que aquí no estamos hablando solamente de la familia como estructura, sino también de sus contenidos: el tipo de interacciones, formas de comunicación, sus expectativas y tradiciones, los mitos y secretos, la religión, sus creencias, las tendencias políticas, sus relaciones sociales, los hábitos y costumbres, el folclore, los valores morales y los intereses que les motivan, sean estos académicos, estéticos, laborales, etc. Todos estos contenidos culturales son interiorizados en virtud de los procesos de socialización que se establecen en el seno de la familia, donde se teje una trama de relaciones sistémicas (p. 44).

En síntesis, se puede afirmar que a través de este estudio fue posible reconocer de la propia voz de las participantes las principales problemáticas que se enfrentan en la actualidad como madres de familia al momento de establecer patrones de crianza en su familia basados en estrategias de crianza de su red familiar de origen y fortalecidas transversalmente por la cultura que cohabitan, las cuales sí influyen en el moldeamiento de estilos de crianza de riesgo para los adolescentes, cuando son asumidas y ejercidas de forma inadecuada y sin considerar el contexto actual y las necesidades esenciales de sus hijos según sus características de personalidad.

En la misma línea, se concluye que los estilos de crianza adoptados por las madres de familia tienen su base en experiencias de vida en su red familiar primaria, lo que les ha generado dificultades al momento de establecer los límites, normas y valores en sus hijos adolescentes, evidenciando repercusiones negativas en sus relaciones sociofamiliares, donde sobresalen aspectos como la desobediencia, la agresividad, las amenazas, las mentiras, entre otros. Esta situación afecta considerablemente las relaciones parentales y, por consiguiente, los lazos afectivos en la red familiar.

Por otro lado, en el adolescente genera distorsión de sus patrones comportamentales ante el espacio de interacción sociofamiliar; no sabe o no comprende de qué manera puede actuar o comportarse; es decir, cuando figuras de autoridad, padre o madre, presentan patrones de crianza inestables, generan en los hijos estados emocionales y comportamentales ambiguos e inestables, incluso pérdida de identidad.

Finalmente, se considera que las actitudes maternales en las prácticas de crianza son determinantes, ya que poseen implicaciones en relación con el proceso de aculturación, considerando el conocimiento del contexto cultural que las identifica, teniendo en cuenta las tradiciones, creencias e ideologías que han sido arraigadas de generación en generación. 


\section{Referencias bibliográficas}

Capano, A., y Ubach, A. (2013). Estilos parentales, parentalidad positiva y formación de padres. Ciencias Psicológicas, 7(1), 83-95. Recuperado de http://www.scielo.edu. uy/pdf/cp/v7n1/v7n1a08.pdf.

Cuervo, A. (2010). Pautas de crianza y desarrollo socioafectivo en la infancia. Diversitas: Perspectivas en Psicología, 6(1), 111-121. Recuperado de http://www.redalyc.org/pdf/679/67916261009.pdf.

de León, B. (2011). La relación familia-escuela y su repercusión en la autonomía y responsabilidad de los niños. Recuperado de http://extension.uned.es/archivos_publicos/webex_actividades/4440/larelacionfamiliaes cuelaysurepercusionenlaautonomiay.pdf.

Diez, A. (2017). Más sobre la interpretación (II): Ideas y creencias. Revista de la Asociación Española de Neuropsiquiatría, 37(131), 127-143. Recuperado de: http://scielo.isciii.es/scielo.php?script=sci_arttext\&pid=S0211$57352017000100008 \& \operatorname{lng}=e s \& t \operatorname{lng}=e s$.

Gallego, T. (2012). Familias, infancias y crianza: Tejiendo humanidad. Revista Virtual Universidad Católica del Norte, 35, 63-82. Recuperado de http://www.redalyc.org/pdf/1942/194224362005.pdf.

Hamui-Sutton, A., y Varela-Ruiz, M. (2013). La técnica de grupos focales. Investigación en Educación Médica, 2(1), 55-60. Recuperado de http://www.scielo.org.mx/ pdf/iem/v2n5/v2n5a9.pdf.

Huamán, K. E. (2016). Estilos parentales e indicadores de salud mental adolescente. Temática Psicológica, 12(1), 35-46. Recuperado de: http://revistas.unife.edu.pe/index.php/tematicapsicologica/article/view/1071

Ibáñez, E. J., Vargas, J. J., y Linares, B. (2017). Evaluación de la diferenciación: Estado actual de la Investigación. Revista Electrónica de Psicología Iztacala, 20(2), 718745. Recuperado de https://repositorio.unam.mx/contenidos/45063.

Infante, A., y Martínez, J. F. (2016). Concepciones sobre la crianza: El pensamiento de madres y padres de familia. Liberabit, 22(1), 31-41. Recuperado de http://www. scielo.org.pe/scielo.php?script=sci_arttext\&pid=S1729-48272016000100003\& lng=es\&tlng=es.

Laca, F. A. (2005). Actitudes y comportamientos en las situaciones de conflicto. Enseñanza e Investigación en Psicología, 10(1), 117-126. Recuperado de https://www. redalyc.org/articulo.oa?id=292/29210108

Mella, O. (2000). Grupos focales ("Focus Groups"): Técnica de investigación cualitativa. Santiago de Chile: CIDE. (Documento de trabajo, 3). Recuperado de https://studylib.es/doc/5135309/grupos-focales-t\%C3\%A9cnica-de-investigaci $\%$ C3\%B3n-cualitativa. 
Mula, A., y Navas, L. (2013). Las actitudes ante la inmigración en los adolescentes y en los jóvenes. Alicante: ECU.

Observatorio de Políticas de las Familias. (2015). Tipologías de familias en Colombia: Evolución 1993-2014. Bogotá: Departamento Nacional de Planeación. (Documento de Trabajo, 2016-1). Recuperado de: https://observatoriodefamilia.dnp.gov.co/ Documents/Documentos\%20de\%20trabajo/D3-tipologias-evolucion_dic3-

(1).pdf.

Organización Panamericana de la Salud. (2009). Manual familias fuertes: Guía para el facilitador. Washington: OPS. Recuperado de https://www.paho.org/hq/dmdocuments/2010/Manual\%20Familias\%20Fuertes\%20Guia\%20para\%20el\%20Facilitador.pdf.

Pulido, S., Castro-Osorio, J., Peña, M., y Ariza-Ramírez, D. P. (2013). Pautas, creencias y prácticas de crianza relacionadas con el castigo y su transmisión generacional. Revista Latinoamericana de Ciencias Sociales, Niñez y Juventud, 11(1), 245-259. Recuperado de: http://www.scielo.org.co/pdf/rlcs/v11n1/v11n1a17.pdf.

Ramírez, M., A. (2005). Padres y desarrollo de los hijos: Prácticas de crianza. Estudios pedagógicos (Valdivia), 31(2), 167-177. Recuperado de https://dx.doi.org/ 10.4067/S0718-07052005000200011

Reyes, L. (2007). La teoría de la acción razonada: Implicaciones para el estudio de las actitudes. Recuperado de: http://www.alfaguia.org/alfaguia/files/1320437914_40.pdf

Rodríguez, M. (2014). La teoría familiar sistémica de Bowen: Avances y aplicación terapéutica. Madrid: McGraw-Hill.

Rosabal-Coto, M. (2012). Creencias y prácticas de crianza: El estudio del parentaje en el contexto costarricense. Revista Costarricense de Psicología, 31(1-2), 65-100. Recuperado de https://www.redalyc.org/articulo.oa?id=4767/476748710006.

Saavedra, V. (2018). Creencias de los padres acerca de la crianza de sus hijos de 4 años de la I.E. Vallesol-Piura. (Tesis de Licenciatura en Educación). Universidad de Piura, Facultad de Ciencias de la Educación. Piura, Perú. Recuperado de: https://pirhua.udep.edu.pe/bitstream/handle/11042/3682/EDUC_068.pdf?sequence $=1$ \&isAllowed=y.

Salamanca, A., y Crespo, C. (2007). El muestreo en la investigación cualitativa. Recuperado de: http://www.sc.ehu.es/plwlumuj/ebalECTS/praktikak/muestreo.pdf.

Sánchez, J. C. (2013). Familias en conflicto: Cómo prevenir, detectar y actuar. Madrid: Mestas Ediciones.

Sánchez, L. (2011). La relación familia-escuela y su repercusiòn en la autonomia y responsabilidad de los niños. Educación Diferencial, Educación Popular, 4-5. Recuperado de http://extension.uned.es/archivos_publicos/webex_actividades/4440/ larelacionfamiliaescuelaysurepercusionenlaautonomiay.pdf 
Torio, S., Peña, J. V., y Rodríguez, M. C. (2008). Estilos educativos parentales: Revisión bibliográfica y reformulación teórica. Teoría de la Educación. Revista Interuniversitaria, 20, 151-178. Recuperado de: https://gredos.usal.es/bitstream/ handle/10366/71805/Estilos_educativos_parentales_revision_b.pdf;jsessionid=EB7C6F10C7D73DDAFC59D88BA361D1F4? sequence $=1$

Torres, L., Garrido, A., Reyes, A., y Ortega, P. (2008). Responsabilidades en la crianza de los hijos. Enseñanza e Investigación en Psicología, 13(1), 77-89. Recuperado de: http://www.redalyc.org/pdf/292/29213107.pdf 